\title{
TAGUNG
}

\section{Die Zusammenarbeit der Polizei in Europa: rechtlicher Rahmen, Forschungsstand und Perspektiven}

\author{
Frédéric Krumbein und Julian Plottka*
}

Hartmut Aden führte in die Konferenz ein und stellte die Leitfragen der Konferenz vor. Auf der Konferenz solle diskutiert werden, inwiefern der Vertrag von Lissabon die polizeiliche Zusammenarbeit in der Europäischen Union ändere und wie der Forschungstand zur Kooperation der Polizei in Europa sei. Weiter werde untersucht, wie die Zusammenarbeit der Polizeibehörden in Europa die Ausbildung und die Professionalität der Polizisten beeinflusse und welchen Einfluss umgekehrt Unterschiede in der Ausbildung und Berufsausübung der Polizisten auf die polizeiliche Kooperation hätten.

Weitere wichtige Punkte, welche die Konferenz beleuchten werde, seien das Spannungsfeld zwischen einer kritischen Erforschung der polizeilichen Arbeit und dargestellten Erfolgen in der polizeilichen Zusammenarbeit, die Berücksichtigung der menschenrechtlichen Perspektive, der Informationsaustausch zwischen Polizeibehörden und die Unterschiede in der Kooperation mit Polizeibehörden innerhalb und außerhalb der Europäischen Union.

Der Vertrag von Lissabon als Quantensprung in der polizeilichen Zusammenarbeit

Cyrille Fijnaut ordnete die polizeiliche $\mathrm{Zu}$ sammenarbeit in Europa in einen größeren historischen Kontext ein. Die erste intensivere Zusammenarbeit von Polizeibehörden habe im 19. Jahrhundert begonnen. Die russische Polizei in der Zarenzeit habe beispielsweise schon ein Büro in Paris eröffnet, um Oppositionelle und Anarchisten zu bekämpfen.

\section{Conference: Police Cooperation in the EU under the Treaty of Lisbon - Opportunities and Limitations}

Tagung der Hochschule für Wirtschaft und Recht, Berlin, in Zusammenarbeit mit dem Arbeitskreis Europäische Integration e.V. und der Groupe Européen de Recherche sur les Normativités

\section{Mit freundlicher Unterstützung der} Europäischen Kommission

Berlin, 21.-22. Februar 2013

Welcome address

Prof. Dr. Bernd REISSERT, President, Hochschule für Wirtschaft und Recht, Berlin

Prof. Dr. Hartmut ADEN, Hochschule für Wirtschaft und Recht, Berlin

Police Cooperation under the Treaty of Lisbon: What is new?

Chair

Prof. Dr. Hartmut ADEN, Hochschule für Wirtschaft und Recht, Berlin

Revolution or Evolution Through the Treaty of Lisbon: Police Cooperation in Europe in the Broader Historical Context

Prof. Dr. Cyrille FIJNAUT, Universiteit Tilburg

Maastricht: The Legal Framework of Police Cooperation in the EU Since and Before the Treaty of Lisbon compared

Prof. Dr. Daniela HEID, European Institute of Public Administration, Maastricht

Recent Developments in Police Cooperation in the EU - the Stockholm Programme and the Treaty of Lisbon

Daniela KIETZ, Stiftung Wissenschaft und Politik, Berlin

* Dr. Frédéric Krumbein, Geschäftsführer des Arbeitskreises Europäische Integration, Berlin. Julian Plottka, Wissenschaftlicher Mitarbeiter am Institut für Europäische Politik, Berlin. 
Gefahren der polizeilichen Zusammenarbeit aus Sicht des demokratischen Rechtsstaats bestünden in dem Prinzip der Geheimhaltung, welches oft polizeilicher Zusammenarbeit zugrunde liege, und der Gefahr eines Missbrauchs der internationalen Polizeikooperation durch Diktaturen. So sei Interpol während des Zweiten Weltkriegs von der NS-Diktatur missbraucht worden, um die repressive nationalsozialistische Herrschaft zu stützen.

Auf Ebene der Europäischen Union sei es überraschend gewesen, dass die polizeiliche $\mathrm{Zu}$ sammenarbeit nach den Reformen durch den Vertrag von Maastricht eine so wichtige Rolle gespielt habe. Diese Vertragsreform habe es nicht unbedingt erwarten lassen.

Der Vertrag von Lissabon stellte schließlich einen Quantensprung im Bereich der polizeilichen Zusammenarbeit dar: Die Kooperation im Bereich der Justiz- und Innenpolitik sei nun eine geteilte Kompetenz der Union und der Mitgliedstaaten. Entscheidungen würden inzwischen in den meisten Fällen mit qualifizierter Mehrheit getroffen. In den letzten Jahren absorbiere aber die Finanz- und Wirtschaftskrise viel politische Kraft, sodass weniger Zeit und Energie zur Verfügung stünden, um die Neuerungen des Primärrechts im Bereich der polizeilichen Zusammenarbeit vollständig umzusetzen.

Daniela Heid referierte über die Rolle der Innen- und Justizpolitik in den EU-Verträgen. Im Vertrag von Amsterdam von 1999 sei die Kooperation im Bereich der Innen- und Justizpolitik überarbeitet worden. Zum ersten Mal sei hier der Begriff des Raums der Freiheit, der Sicherheit und des Rechts in der Europäischen Union verwandt worden. Die Schaffung und Entwicklung dieses Raums stellt seitdem ein Ziel der Europäischen Union dar. Art. 30 des Vertrags über die Europäische Union in der Fassung des Vertrags von Amsterdam habe außerdem die Zusammenarbeit der Polizei beinhaltet und unter anderem die Speicherung und den Austausch von Daten und Informationen umfasst.
Differentiated Integration: Ever More Relevant for Police Cooperation After the Treaty of Lisbon?

Dr. Funda TEKIN, Universität zu Köln/Institut für Europäische Politik, Berlin

Policing in Europe: Increasing Professionalism? Chair

Prof. Dr. Sabrina SCHÖNROCK, Hochschule für Wirtschaft und Recht, Berlin

Police Training and Police Studies in the EU Member States: Towards higher Standards by Coordination?

Prof. Dr. Hans-Gerd JASCHKE, Hochschule für Wirtschaft und Recht, Berlin

Has Police Cooperation in the EU Become More Professional in Recent Years?

Prof. Dr. Monica DEN BOER, Nederlandse Politieacademie/Vrije Universiteit Amsterdam

Empirical Insights into Police Cooperation in the EU

Chair

Lars OSTERMEIER, Technische Universität Berlin

What Do We Know About Police Cooperation in the EU - the State of Empirical Research

Dr. Wilhelm KNELANGEN, Christian-AlbrechtsUniversität zu Kiel

European and International Cooperation in Practice: The Case of the Berlin Police

Bettina RAUCH-SCHULZ, Head of EU Unit/International Projects (LKA 74), Landeskriminalamt, Berlin

Do Council Instruments Matter? The Effect of Council Instruments on Practical Police Cooperation in the $E U$

Dr. Ludo BLOCK, ARC Centre of Excellence in Policing and Security (CEPS), Griffith University, Brisbane

Collaborative Work Between Police Forces: Knowledge Sharing Capabilities and Best Practices Using the Example of Cross-border German-Polish Police and Customs Cooperation Centre in Świecko (Poland)

Natalie HIRSCHMAN/Mario GRUSCHINSKE (with Stefanie GILJOHANN and Susanne STEINMÜLLER), Institut für Polizeiwissenschaft, COMPOSITE (Comparative Police Studies in the EU), Fachhochschule der Polizei des Landes Brandenburg, Oranienburg 
Durch den Vertrag von Lissabon sei die DreiSäulen-Struktur der Europäischen Union aufgegeben worden. Fast alle Bereiche in der polizeilichen und justiziellen Zusammenarbeit fielen in den Bereich der qualifizierten Mehrheitsentscheidungen. Großbritannien, Dänemark und Irland seien kein Teil der Zusammenarbeit in diesem Bereich (sie hätten ein ,Opt-out' für diesen Bereich), aber verfügten über die Option jederzeit Teil des Raums der Freiheit, der Sicherheit und des Rechts zu werden.

Der Europäische Gerichtshof werde ab dem 1. Dezember 2014 über die vollständige (bis dahin eingeschränkte) Kompetenz verfügen, Urteile im Bereich der Kooperation in der EUInnen- und Justizpolitik zu fällen. Allerdings werde er weiterhin nicht die Kompetenz besitzen, die Verhältnismäßigkeit oder die Gültigkeit von polizeilichen Operationen zu prüfen und zu bewerten.

Die Neuerungen des Vertrags von Lissabon in der Zusammenarbeit im Bereich der Innenund Justizpolitik wurden ebenfalls von Daniela Kietz diskutiert. Sie sprach über die Normalisierung der Entscheidungsfindung durch den Vertrag von Lissabon und die Einführung der qualifizierten Mehrheit. Weiterhin gebe es zunehmend eine Diskussion über die richtige $\mathrm{Ba}-$ lance zwischen der verstärkten Kooperation im Sicherheitsbereich und der Garantie von Bürger- und Menschenrechten.

Vor der Unterzeichnung des Vertrags von Lissabon habe es verschiedene Kritikpunkte an der polizeilichen Zusammenarbeit in der Europäischen Union gegeben, wie eine undemokratische Entscheidungsfindung, ein kompliziertes System von ,Opt-outs', die Zusammenarbeit von Staaten außerhalb der EU-Institutionen, eine begrenzte Effektivität und Umsetzung beschlossener Kooperationsschritte im Vergleich zu den ambitionierten Zielen und Plänen sowie ein zu stark sicherheitszentrierter Ansatz in der Polizeizusammenarbeit. Die künftigen Herausforderungen lägen vor allem in einer besseren Implementierung von be-
Towards a Higher Standard of Civil Liberties by Police Cooperation? The Story of Dialogue Policing in Europe and the Ongoing EU Project on Good Practice for Dialogue and Communication as Strategic Principles for Policing Political Manifestations in Europe (GODIAC)

Daniel BRUNSCH, Universität zu Köln

Police Cooperation in the EU and beyond: Integrating Third Countries into Police Cooperation under the Treaty of Lisbon Chair

Prof. Dr. Claudius OHDER, Hochschule für Wirtschaft und Recht, Berlin

Contributions of Member States to EU Police External Operations

Dr. Olivier CAHN, Université de Cergy-Pontoise

EU Police Cooperation with, and Assistance to, Police in the Wider European Neighbourhood

Liam O'SHEA, University of St. Andrews

The Exchange of Information between Police Organisations in the $\mathbf{E U}$

Chair

Prof. Dr. Clemens ARZT, Hochschule für Wirtschaft und Recht, Berlin

The Expansive Trend of Exchanging Information Between European Police Organisation - Reasons and Phenomena

Prof. Dr. Hartmut ADEN, Hochschule für Wirtschaft und Recht, Berlin

The Exchange of Police Information in the EU Under the Treaty of Lisbon-Routine and Difficulties from a Practical Perspective

Michael NIEMEIER, Director International Coordination, Bundeskriminalamt, Berlin

Conflicts Between Data Protection Harmonisation and a High Level of Protection: The Draft Directive on the Protection of Individuals with Regard to the Processing of Personal Data [...] for the Purposes of Prevention, Investigation, Detection or Prosecution of Criminal Offences [...]

Peter SCHAAR, Bundesbeauftragter für den Datenschutz und die Informationsfreiheit, Bonn

Civil Liberties and the Exchange of Information between Law Enforcement Agencies: the European Parliament's Role

Jan-Philipp ALBRECHT, MEP, Member of the Civil Liberties, Justice and Home Affairs Committee (LIBE), Strasbourg/Brussels 
schlossenen Maßnahmen im Bereich der polizeilichen Zusammenarbeit und einer Garantie der Bürger- und Menschenrechte der in Europa lebenden Menschen.

Funda Tekins Vortrag beschäftigte sich mit differenzierter Integration im Bereich der Innen- und Justizzusammenarbeit. Differenzierte Integration meine, dass eine Gruppe von Staaten anderen Regeln unterworfen sei als eine andere Gruppe. Möglichkeiten einer differenzierten Integration bestünden in Form einer verstärkten Zusammenarbeit einiger Staaten innerhalb der EU-Strukturen, in Form von ,Opt-outs' ${ }^{6}$ und ,Opt-ins' einzelner Staaten in einem Politikfeld oder in Form einer vertieften Zusammenarbeit von EU-Mitgliedstaaten außerhalb der EU-Strukturen.

In diesem Politikfeld erkläre sich eine differenzierte Integration unter anderem aus der Rolle der Innen- und Justizpolitik als einem Kernbestandteil der souveränen Nationalstaaten. Aus diesem Grund bewahrten die Nationalstaaten vielfach ein Vetorecht in diesem Bereich und votierten beispielsweise für ,Optouts'.

Mitgliedstaaten präferierten lange Zeit, Kooperationsvereinbarungen außerhalb der EUStrukturen zu schließen, wie beispielsweise das Schengen-Abkommen. Erst später sei dieses in die EU-Struktur, mit ,Opt-outs' für einige Staaten, integriert worden. Großbritannien verfüge im Bereich der Innen- und Justizpolitik über die Möglichkeit, Opt-outs` in Anspruch zu nehmen, aber ebenfalls in einzelnen Bereichen für ein ,Opt-in' zu votieren und damit ,cherry-picking“ zu betreiben.

Die Konsequenzen der verschiedenen ,Optouts' bestünden vor allen in einer komplizierten europäischen Struktur in der Innen- und Justizpolitik für alle beteiligten Staaten und einem Verlust von nationalem Einfluss und von Agendasetzungsmacht für die, Opt-out'-Staaten bei gleichzeitiger Bewahrung ihrer nationalen Souveränität.
Europol Joint Supervisory Body: Coordinated Data Protection for Police Cooperation in the EUOutcome and Limitations

Gabriele LÖWNAU, Member of the Europol Joint Supervisory Body/Head of Unit, Bundesbeauftragter für den Datenschutz und die Informationsfreiheit, Bonn

Cross-border Law Enforcement Cooperation with the Private Sector \& the EU Data Protection Legal Framework

Gertjan BOULET, Vrije Universiteit Brussel

Round-table discussion: The Future of Police Cooperation in the $\mathbf{E U}$

Chair

Prof. Dr. Hartmut ADEN, Hochschule für Wirtschaft und Recht, Berlin

Participants

Jan-Philipp ALBRECHT, MEP, Member of the Civil Liberties, Justice and Home Affairs Committee (LIBE), Strasbourg/Brussels

Prof. Dr. Monica DEN BOER, Nederlandse Politieacademie/Vrije Universiteit Amsterdam

Michael NIEMEIER, Director International Coordination, Bundeskriminalamt, Berlin

Bettina RAUCH-SCHULZ, Head of EU Unit/International Projects (LKA 74), Landeskriminalamt, Berlin

Detlef SCHROEDER, Deputy Director, European Police College - CEPOL, Bramshill

\section{Closing remarks}

Prof. Dr. Hartmut ADEN, Hochschule für Wirtschaft und Recht, Berlin

Fortschritte in der Polizeiarbeit durch die Zusammenarbeit in Europa

Hans-Gerd Jaschke sprach in seinem Vortrag über die aktuelle Situation der Polizeistudien und deren Verhältnis zur Polizeiausbildung in Europa. Kulturelle und strukturelle Unterschiede zwischen der Polizei vieler südeuropäischer Staaten und den nördlichen Staaten beeinflussten die jeweilige Ausbildung der Polizei. In den nördlichen Staaten diene die Poli- 
zei vornehmlich den Bürgern. In den südlichen EU-Ländern beschütze die Polizei in erster Linie den Staat und seine Verfassung.

Weiterhin hätten die Bologna-Reformen die Polizeiausbildung nachhaltig beeinflusst. Eine Umfrage über die Umsetzung der Bologna-Reformen in Polizeiakademien und -hochschulen habe ergeben, dass 22 EU-Mitgliedstaaten inzwischen bolognaakkreditierte Polizeistudiengänge mit Bachelor- und Masterabschlüssen besäßen. Ausnahmen bildeten Dänemark, Großbritannien, Luxemburg, Slowenien und Zypern.

Seit mehr als zehn Jahren befinde sich die wissenschaftliche Erforschung der Polizei auf dem Vormarsch. Aber es gebe unterschiedliche Auffassungen bei Praktikern und Wissenschaftlern. Viele Praktiker meinten, sie bräuchten keine Forschung über ihre eigene Arbeit als Polizisten. Eine entscheidende Frage der Polizeiwissenschaften bestünde außerdem in dem kritischen oder nichtkritischen Verhältnis zu ihrem Untersuchungsgegenstand: Macht die Polizeiwissenschaft Forschung für die Polizei oder über die Polizei?

Die Hauptthemen der Polizeistudien seien von den 1960er bis 1990er Jahren vor allem die Anwendung von Gewalt durch die Polizei, das Verhältnis zwischen Bürgern und Polizisten, die Befugnisse der Polizei, die Polizeikultur, die Effektivität der polizeilichen Arbeit und die Überwachung der Bürger durch die Polizei gewesen.

Insgesamt bestünde eine Kluft zwischen Forschung und Praxis. Nur wenige Polizisten griffen die Ergebnisse der Forschung über die Polizei in ihrer Arbeit auf. Positiv sei eine stärkere transnationale Kooperation in Europa im Bereich der Polizeistudien. Der Bologna-Prozess werde von den Polizeihochschulen zunehmend akzeptiert. Hindernisse bestünden aber in zu wenigen Teilnehmern an den Bildungsangeboten auf europäischer Ebene, einem zu gerin- gen Austausch zwischen Studierenden und Lehrenden und in sprachlichen Problemen, die den Austausch behinderten.

Monica den Boer referierte über die zunehmende Professionalisierung der Polizei in Europa. Die Fragestellung ihres Vortrags lautete: Wie beeinflusst Europa die Professionalisierung von Polizisten? Der europäische Einfluss sei dabei positiv zu bewerten. Die Polizisten lernten voneinander, reflektierten kritischer über die eigene nationale Politik und sie erhielten neue Ideen aus Europa.

Für Polizisten sei es in ihrer Ausbildung nicht erforderlich, die gesamte Struktur europäischer Institutionen, zum Beispiel den Europarat, zu kennen. Das gleiche gelte für die Gesamtheit der Institutionen und Politiken der Europäischen Union. Nichtsdestotrotz müssten Polizeibeamte in gehobenen Positionen in der Europäischen Union über grundlegende Kenntnisse der europäischen Regierungsstrukturen und der europäischen Behörden im Bereich der Innen- und Justizpolitik verfügen, wie beispielsweise über Europol oder Frontex ${ }^{1}$. Darüber hinaus seien Kenntnisse anderer europäischer Sprachen und Kulturen wichtig.

Alle Polizisten sollten zudem einem Ethikcodex folgen und müssten dafür die maßgeblichen menschenrechtlichen Grundlagen kennen, wie die Charta der Grundrechte der Europäischen Union und die Europäische Menschenrechtskonvention. Außerdem müssten sie einen Überblick über die spezifischen Dokumente für ihre eigene Arbeit haben, wie den „UN Code of Conduct for Law Enforcement Officials" oder den Ethikcodex des Europarates.

Defizite im Bereich der Professionalisierung von Polizisten in Europa bestünden unter anderem in einer zu geringen Kooperation der Polizisten in Europa und einer starken Fragmentierung und Diversität des Niveaus der polizeilichen Professionalität in den verschiedenen Mitgliedstaaten der Europäischen Union.

1 Europäische Agentur für die operative Zusammenarbeit an den Außengrenzen. 
In der anschließenden Diskussion über die Polizeiwissenschaften in Europa und ihre aktuellen Herausforderungen wurde unter anderem über die Gewalt von Polizisten und den Selbstmord von Polizisten gesprochen, die beide sehr sensible Themen darstellten und wenig erforscht seien. So würden etwa 95 Prozent aller Fälle von Polizeigewalt, die vor Gericht landeten, abgewiesen.

\section{Wirkungsanalysen politischer Entscheidun- gen: ein Forschungsdesiderat}

Das der empirischen Forschung zur Polizeikooperation gewidmete Panel umfasste Beiträge von Wissenschaftlern und von Praktikern der Polizeiarbeit. Damit sollte ein Bias der Forschung zur polizeilichen Zusammenarbeit überbrückt werden, den Wilhelm Knelangen in seinem Überblick über den aktuellen Stand der Forschung herausarbeitete. Während die politischen Entscheidungsprozesse im Politikfeld der polizeilichen Kooperation inzwischen gut erforscht seien, fehlten Arbeiten, die sich mit der Wirkung dieser Entscheidungen auf die Polizeiarbeit befassten. Bei dem Forschungsfeld handele es sich nach zehn Jahren intensiver Forschungsarbeit immer noch um eine „Wachstumsindustrie“. Zuvor habe ihm die Wissenschaft trotz seiner besonderen Dynamik in der europäischen Integration lange skeptisch gegenüber gestanden.

Eine solche zuvor geforderte Betrachtung des Einflusses der Politik auf die tägliche Polizeiarbeit unternahm Bettina Rauch-Schulz aus der Perspektive des Berliner Landeskriminalamtes. Auf Basis eines Überblicks über Maßnahmen der Polizeikooperation in der Berliner Polizei identifizierte sie Ursachen für die noch unzureichende Kooperation im Alltag. Diese seien sowohl auf Seiten der Entscheidungsträger (Budgetkürzungen, Personalmangel) als auch der Polizeibeamten (fehlende Sprachkenntnisse, zögerlicher Kooperationsbereitschaft) zu finden, genauso wie es Probleme bei der Umsetzung gebe (fehlende Netzwerke, Schwierigkeiten bei der Strategieerarbeitung).
Auch drei weitere präsentierte Forschungsarbeiten waren dem von Knelangen identifizierten Desiderat gewidmet: Während eine quantitative Studie die Effektivität von Ratsbeschlüssen untersuchte und damit dem Zusammenhang von politischer Ebene und alltäglicher Polizeiarbeit gewidmet war, konzentrierten sich eine Einzelfallstudie zur deutsch-polnischen Polizei- und Zollkooperation sowie eine international vergleichende Studie zum Umgang der Polizei mit Demonstrationen auf die Analyse des Wandels der Polizeiarbeit.

Ludo Block analysierte 137 Ratsbeschlüsse aus den Jahren 1995 bis 2004, die als Instrumente im Bereich der Polizeikooperation zu qualifizieren seien, auf einen Zusammenhang zwischen deren Form (bindende vs. nicht bindende Instrumente) sowie deren ,professional rationality“" (Einbeziehung von Expertenwissen aus der Polizeiarbeit) und ihrer Wirkung. Während er für die Form der Instrumente keinen Einfluss auf deren Wirkung finden konnte, wiesen seine Daten eine signifikant positive Korrelation zwischen der Einbeziehung von Expertenwissen und der Wirkung von Instrumenten auf.

Natalie Hirschmann und Mario Grunschinske präsentierten die Ergebnisse einer Fallstudie zur deutsch-polnischen Polizeikooperation, die Teil eines internationalen Forschungsprojekts in 10 Staaten ist. Auf Basis von Interviews, Dokumentenanalysen, teilnehmenden Beobachtungen sowie Fragebögen werden die Rahmenbedingungen des grenzüberschreitenden Informationsaustausches in der Polizeiarbeit untersucht. Zwischen Deutschland und Polen sei dieser laut der befragten Polizisten aufgrund der engen persönlichen grenzüberschreitenden Kontakte effektiv. Als Hindernisse für einen effektiven Informationsaustausch wurden unterschiedliche nationale Organisationstrukturen und Rechtsrahmen sowie fehlende gemeinsame Datenbanken und eine mangelhafte Öffentlichkeitsarbeit identifiziert.

Daniel Brunsch untersuchte in einer international vergleichenden Studie den Umgang der Polizei mit Demonstrationen und Protesten 
(„crowd management") im mehreren Staaten. Seine Ausgangshypothese war, dass dieses aufgrund einer wachsenden Zahl von grenzüberschreitenden Protestereignissen repressiver geworden sei. In der Untersuchung der Polizeistrategien beim ,crowd management ${ }^{*}$ konnte er jedoch keinen generellen Trend feststellen. Während in drei untersuchten Fällen verstärkt ein präventiver dialogorientierter Ansatz verfolgt werde, werde in vier Ländern eine responsive Strategie angewandt.

\section{Interne und externe Bedingungen von $E U$ - Polizeimissionen in der Analyse}

Nach der Diskussion von Forschungsergebnissen zur Europäisierung der Polizeiarbeit in der Europäischen Union, war das folgende Panel der Einbindung von Drittstaaten in die europäische Polizeikooperation gewidmet. Auch in den Vorträgen über die externe Dimension der EU-Politik zur Polizeikooperation schienen wieder die beiden Analyseebenen des politischen Entscheidungsprozesses und des Wandels in der konkreten Polizeiarbeit auf.

Den Entscheidungsprozessen war der Vortrag von Olivier Cahn gewidmet, der die Relevanz nationaler Interessen und des Primärrechts für Entscheidungen über Polizeimissionen diskutierte. Alle Mitgliedstaaten hätten sich bereits an einer Mission beteiligt, Umfang und Gründe dafür unterschieden sich aber. Eine Gruppe von Mitgliedstaaten engagiere sich mit dem Ziel, die EU-Außenpolitik zu stärken. Eine zweite Gruppe sähe die EU-Polizeimissionen jedoch in Konkurrenz zur entsprechenden Politik der NATO. ${ }^{2}$ Eine dritte Gruppe verhalte sich neutral. Als weitere Gründe für ein Engagement identifizierte Cahn, die generelle Erwartung eines Mindestmaßes an Beteiligung und länderspezifische Interessen, wie im Falle ehemaliger Kolonialmächte. Die letzte Vertragsreform habe die EU-Außenpolitik insgesamt gestärkt ohne sie jedoch zu supranationalisieren. Dieser ausgebliebene primärrechtliche Impuls in Verbindung mit der derzeitigen
Sparpolitik lasse künftig eine weiter abnehmende Anzahl von Missionen erwarten.

Eine vergleichende Evaluation der Wirkung von EU- und von anderen Akteuren geführten Polizeimissionen nahm Liam O'Shea am Beispiel von Kirgisistan und Georgien vor. In beiden untersuchten Fällen habe es Maßnahmen des ,capacity buildings“ jedoch keine Kooperation bei der operativen Polizeiarbeit gegeben. Zentrale Voraussetzungen für den Erfolg von außen beförderter Reformen seien die Rahmenbedingungen innerhalb des Landes: einerseits der Wille zur Reform und andererseits die finanzielle Ausstattung der Polizei. Während dieser Wille in Georgien bestanden hätte und Polizisten dort nicht auf Nebeneinkünfte angewiesen seien, würden Clanstrukturen eine Reform in Kirgisistan verhindern. Fehlten diese beiden Voraussetzungen, so könne auch durch internationale Geldgeber kein Wandel angestoßen werden.

\section{Informationsaustausch: Datenschutz als Herausforderung der Polizeikooperation}

Die Anforderungen des Datenschutzes stehen in einem Spannungsverhältnis zur Notwendigkeit des Informationsaustausches für eine effektive Polizeiarbeit. Hinsichtlich Letzterem konstatierte Hartmut Aden einen expansiven Trend der Sammlung von immer mehr Daten durch die Polizeibehörden. Dies sei heute ein Schlüsselfaktor erfolgreicher Kriminalitätsbekämpfung so Michael Niemeier, was die zunehmende Nutzung und Anzahl von Treffern bei der grenzüberschreitenden Informationsabfrage belegten. Deshalb müsse grundlegendes Prinzip beim Informationsaustausch die Zugänglichkeit von Daten seien. Aden betonte jedoch auch, dass ein zunehmendes Datenvolumen nicht per se eine effektivere Polizeiarbeit bedeute, sondern Fragen des Qualitätsmanagements und der Aktualisierung der Daten immer wichtiger würden. Hier könne Datenschutz sogar einen Beitrag zur Qualität der Daten und damit zur Verbesserung der Polizeiarbeit leisten, so Gabriele Löwnau.

2 North Atlantic Treaty Organization. 
Für Jan Philipp Albrecht sind die Schaffung des Raums der Freiheit, der Sicherheit und des Rechts sowie der Datenschutz gleich wichtige Aufgaben der EU-Politik. Die Polizeikooperation müsse intensiviert und der europäische Datenschutz verbessert werden. Seit der Stärkung seiner Rechte bei der letzten Vertragsreform gebe es mit dem Europäischen Parlament einen Anwalt des Datenschutzes auf EU-Ebene. So sah Albrecht die Datenschutzprobleme auch weniger auf EU-Ebene bei Europol, sondern in der Umsetzung von EU-Standards durch die Mitgliedstaaten.

Mit einem Bericht aus ihrer alltäglichen Arbeit belegte Löwnau die genannte Vermutung mit Blick auf Europol, das weitestgehend die rechtlichen Vorgaben einhalte. Hier bestünden keine ernsthaften Datenschutzprobleme.

Hinsichtlich der Frage des Datenschutzes in den Mitgliedstaaten sah Peter Schaar die Herausforderung anders gelagert als Albrecht. Zwar seien die EU-Standards insgesamt hoch, dennoch bestehe im Falle der Harmonisierung nationaler Standards die Gefahr einer Verwässerung des Datenschutzes. Hier könne es zum Konflikt zwischen europäischem und nationalem Recht kommen. Um diesen zu vermeiden, forderte Schaar statt einer Vollharmonisierung die Schaffung von EU-Mindeststandards über die die Mitgliedstaaten hinausgehen könnten.

Auch Niemeier sah Probleme bei der Umsetzung von EU-Recht in einigen Mitgliedstaaten, jedoch nicht den Datenschutz, sondern die Instrumente des Informationsaustausches betreffend. Neben bestehenden Zeitverzögerungen bei der grenzüberschreitenden Abfrage von Daten, seien die Umsetzungsprobleme ein weiteres Hemmnis für die polizeiliche Zusammenarbeit.

Zwei weitere Herausforderungen für den europäischen Datenschutz wurden kurz angesprochen: Löwnau verwies darauf, dass der Informationsaustausch mit Behörden in Drittstaaten in besonderem Maße Fragen der Anwendbarkeit europäischen Datenschutzrechts aufwirft. Gleiches gilt für die Weitergabe von Informationen an private Akteure im Rahmen der polizeilichen Kooperation, wie Getjan Boulet unterstrich.

Europäische Polizeiträume: Perspektiven der Polizeikooperation

In der abschließenden Diskussion wagten die Teilnehmer einen Ausblick auf die Zukunft der Polizeikooperation. Zentraler von allen Teilnehmern genannter Wunsch war, dass die Politik künftig die Belange der alltäglichen Polizeiarbeit stärker berücksichtige. Michael Niemeier verwies darauf, dass dazu ein besserer Informationsaustausch zwischen den politischen Entscheidungsträgern und der operativ tätigen Ebene notwendig sei, was Jan-Philipp Albrecht auf die Frage nach der Lobby der Polizeibeamten in Brüssel zuspitzte. Er habe den Eindruck, dass die Lobbyisten in Brüssel, die für sich in Anspruch nähmen, die Beamten zu vertreten, sie nicht immer wirklich repräsentierten.

In engem Zusammenhang mit diesem neuen Bezugspunkt kommender EU-Politik stand die Forderung nach einer stärkeren Europäisierung der Polizei. Neben anderen hob Bettina Rauch-Schulz die Bedeutung persönlicher Kontakte und Erfahrungen hervor: Ein Polizist, der Kooperation praktisch erlebt habe, stelle diese nie wieder infrage. Solange Kooperation aber nicht Teil des Alltags jedes Polizisten sei, bestehe weiterhin die Notwendigkeit zur Begründung der Kooperation als Politikziel. Auch Niemeier sah die Umsetzung der politischen Dimension der Kooperation in der alltäglichen Arbeit als Aufgabe für die Polizei.

Mit Blick auf die bisherige Entwicklung des Politikfeldes kritisierte Monica de Boer die fehlende Blaupause. Die Entwicklung sei inkrementell verlaufen und von Aktivismus getrieben gewesen. Für die Zukunft wünschte sie sich, dass dies überwunden werde. Da die Arbeit auf EU-Ebene zunehmend operativ werde, sei deren Kontrolle auf supranationaler Ebene notwendig. Im Bereich des Informationsaus- 
tausches lehnte Rauch-Schulz die Schaffung neuer Institutionen ab. Die bestehenden reichten bei Weitem aus, sie müssten nur stärker genutzt und gegebenenfalls verbessert werden. Niemeier regte konkret eine Evaluation von Europol an.

Ein weiteres mehrfach angesprochenes Thema war die Frage der europäischen Finanzierung der Polizeikooperation. Detlef Schroeder sah die größte Herausforderung für die Polizei darin, dass künftig bessere Arbeit mit weniger Ressourcen geleistet werden müsse. Dazu sei eine bessere Abstimmung zwischen den in der Polizeikooperation aktiven Akteuren (Europäische Union, Interpol, $\mathrm{OSZE}^{3}$ ) sowie den Staaten notwendig, um die Duplizierung von Maßnahmen zu vermeiden. Polizeikooperation müsse auf die Bereiche konzentriert werden, in denen sie einen Mehrwert bringe. Auch Albrecht verwies darauf, dass Prioritätensetzung gleichzeitig eine Entpriorisierung anderer Aufgabenfelder bedeute. Einigkeit schien darin zu bestehen, dass Strafverfolgung eine der kommenden Prioritäten sein werde.

3 Organisation für Sicherheit und Zusammenarbeit in Europa. 